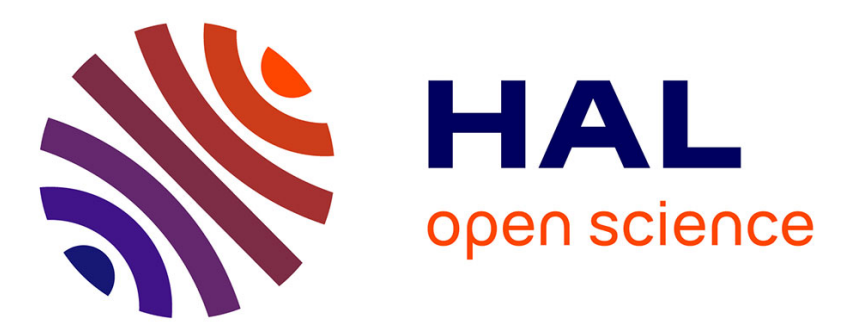

\title{
Adaptive and Warning Displays with Brain-Computer Interfaces : Enhanced Visuospatial Attention Performance
}

\author{
Romain Trachel, Thomas Brochier, Maureen Clerc
}

\section{To cite this version:}

Romain Trachel, Thomas Brochier, Maureen Clerc. Adaptive and Warning Displays with BrainComputer Interfaces: Enhanced Visuospatial Attention Performance. IEEE/EMBS 6th international conference on neural engineering, Nov 2013, San diego, United States. hal-00903288

\section{HAL Id: hal-00903288 \\ https://hal.inria.fr/hal-00903288}

Submitted on 11 Nov 2013

HAL is a multi-disciplinary open access archive for the deposit and dissemination of scientific research documents, whether they are published or not. The documents may come from teaching and research institutions in France or abroad, or from public or private research centers.
L'archive ouverte pluridisciplinaire $\mathbf{H A L}$, est destinée au dépôt et à la diffusion de documents scientifiques de niveau recherche, publiés ou non, émanant des établissements d'enseignement et de recherche français ou étrangers, des laboratoires publics ou privés. 


\title{
Adaptive and Warning Displays with Brain-Computer Interfaces : Enhanced Visuospatial Attention Performance
}

\author{
Romain Trachel $^{a, b}$, Thomas Brochier ${ }^{a}$ and Maureen Clerc $^{b, *}$
}

\begin{abstract}
Brain-Computer Interfaces (BCI) can provide innovative solutions beyond the medical domain. In human research, visuospatial attention is often assessed from shifts in head or gaze orientation. However in some critical situations, these behavioral features can be dissociated from covert attention processes and brain features may indicate more reliably the spatial focus of attention. In this context, we investigate whether EEG signals could be used to enhance the behavioral performance of human subjects in a visuospatial attention task. Our results demonstrate that a BCI protocol based on adaptive or warning displays can be developed to shorten the reaction time and improve the accuracy of responses to complex visual targets.
\end{abstract}

\section{INTRODUCTION}

Visuospatial attention is a selection process that allows for continuously allocating cognitive ressources to specific locations in the environment. Spatial attention can be allocated in the visual field either overtly by gazing at cued locations, or covertly without eye movements. A visual cue briefly displayed in the periphery can produce exogenous covert attention processes that capture the focus of attention, before any visual saccade. Moreover, a visual cue displayed in central vision can produce endogenous covert attention processes to anticipate an upcoming visual event at the spatially cued location. In both situations, the processing of visual targets displayed at attended spatial location is facilitated while it is actively suppressed at remote locations [8], [3]. In addition, the anticipatory covert attention processes reflect cortical inhibitions at unattended spatial locations which could be detected by a power increase in the $\alpha$-band $(8-14 \mathrm{~Hz})$ over posterior brain regions at the ispsilateral side of attention [10].

Active Brain-Computer Interfaces (BCI) are used to control an external device in medical applications using $\alpha$ band activity [11], [12], [1]. Moreover, passive BCI are also used in non-medical applications for monitoring human workload in safety protocols [13]. For neuroergonomics [7], BCI can reveal covert cognitive processes and detect factors increasing the risk of human errors. During vehicle control, the operator continuously gazes with central vision while anticipating events that could appear in the environment. In a complex situation, intelligent dashboards could optimize the user's behavioral performance by adapting visual information in locations where spatial attention is engaged. Actually, the brain activity could provide valuable information about

\footnotetext{
* Contact author maureen.clerc@sophia.inria.fr

a Aix-Marseille Université, INT - CNRS UMR 7289, Marseille, France.

${ }^{b}$ Athena Project-Team, INRIA Sophia Antipolis - Méditerranée , Sophia Antipolis, France
}

covert processes, producing more rapid and accurate behavioral responses through active and passive BCIs. Therefore, we propose a BCI for enhancing visuospatial attention performance in a complex visuospatial attention task inducing spatial errors in anticipation of a target's location. The BCI performance is evaluated in human subjects over 2 online sessions in which the BCI reduces spatial errors and subsequent impaired reactions, either by adaptively displaying the target at an attended location, or by warning the subject before the target is displayed at unattended location. The subject's spatial errors, reaction speed and accuracy are compared between the offline calibration session and the online session.

\section{EXPERIMENTAL PROCEDURE}

\section{A. Subjects and Materials}

5 naive BCI subjects (male, aged between 20 and 35) with normal or corrected-to-normal vision participated in the experiment. Brain activity was recorded by ElectroEncephaloGraphy (EEG) using the BioSemi ActiveTwo system ${ }^{1}$ with 64 sensors sampled at $2048 \mathrm{~Hz}$. Eye movements were monitored by ElectroOculoGraphy (EOG) with bipolar electrodes measuring voltage differences between the outer canthus of each eye (horizontal component) and between the lower right eye and the Fp2 electrode (vertical component). The OpenViBE software [9] was used for real-time signal processing, feature extraction and classification. The task was designed in Matlab using the PsychToolbox ${ }^{2}$ for visual display and the Data Aquisition toolbox for recording behavioral responses. BCI calibration was performed offline on a High Performance Computing (HPC) cluster using the $\mathrm{MNE}^{3}$ and sklearn ${ }^{4}$ Python toolboxes for EEG processing and feature classification.

\section{B. Visuospatial Attention Task}

Subjects sat comfortably $60 \mathrm{~cm}$ away from a 20' CRT monitor (resolution $800 \times 600,120 \mathrm{~Hz}$ ) and performed a visuospatial attention task based on Posner's paradigm [8]. In this task, we used a complex spatial cue (SC) in order to shift attention towards one of two opposite target locations in the periphery. Subjects were instructed to sustain attention at the location precued by the SC, while continuously fixating a central point. Following a random delay of 1.5 to $2.5 \mathrm{~s}$ after SC, they had to detect and identify a visual target (VT)

\footnotetext{
${ }^{1}$ http://www.biosemi.com

${ }^{2}$ http://psychtoolbox.org

${ }^{3}$ http://martinos.org/mne/mne-python.html

${ }^{4}$ http://scikit-learn.org
} 
briefly flashed $(70 \mathrm{~ms})$ at precued location, using a response box with two switches pressed by left or right thumb after VT onset. Each location was displayed in the lower left or right visual field, at $\pm 9.23^{\circ}$ horizontal and $-5^{\circ}$ vertical eccentricity from the center [2].

The SC consisted of randomly moving dots displayed for $250 \mathrm{~ms}$ around the central fixation point. The dot directions were drawn at every trial from a Von-Mises distribution whose mean indicates the upcoming target location and variance is the inverse of motion coherence. The mean direction was difficult to identify with low motion coherence, producing high spatial error rate (SER) of attention shifts. Therefore, we used an adaptive procedure [14] to set a coherence threshold that ensures SER would be less than $10 \%$ and defined two categories of SC :

- The predictive SC with coherence set at threshold.

- The ambiguous SC with coherence set at 0.

These coherence settings were used to induce higher SER with ambiguous than predictive SC. Subjects were not aware of these settings and could not distinguish the two categories because SC were briefly displayed and randomly interleaved from trial to trial.

The VT consisted in a Gabor pattern tilted at $\pm 45^{\circ}$ and embedded in a dynamic noise [3] continuously displayed at both target locations. The noise contrast was $35 \%$ of maximum intensity with a spatial resolution 10 times lower than the VT. The VT contrast was set below the noise contrast to produce high error rate (ER) and reaction time (RT) if VT were not displayed at attended location. Therefore, we used the adaptive procedure to ensure ER would be less than $10 \%$ at attended location. The target locations were balanced and followed a pseudo random sequence. Subjects were instructed to respond as accurately and as fast as possible after detecting the VT by pressing the right (resp. left) switch to identify VT tilted at $+45^{\circ}$ (resp. $-45^{\circ}$ ). The response was followed either by a positive feedback or by an error message. Subjects reported if the VT had appeared at the attended or unattended location, then the next trial started.

\section{Recording Sessions}

Before the experiment, subjects were trained for 52 trials and performed the 2 adaptive procedures to set SC coherence and VT contrast. Each adaptative procedure lasted 40 trials or more until convergence. Then EEG signals and behavioral responses were recorded over 3 consecutive sessions:

- The offline session, used to evaluate the behavioral performance baseline and to calibrate the BCI over 4 blocks of 52 trials.

- The $1^{\text {st }}$ online session, used to evaluate behavioral enhancement by BCI-based target displays over 4 blocks of 52 trials.

- The $2^{\text {nd }}$ online session, used to evaluate behavioral enhancement by BCI-based warning displays over 2 blocks of 52 trials.

\section{METHODS}

\section{A. Signal Processing}

EEG signal processing and feature extraction are performed online using the OpenViBE software. EOG signals are filtered to $1-10 \mathrm{~Hz}$ and analyzed a posteriori to reject trials contaminated by eye movements, occuring between the cue and the target display. For each trial, we set an amplitude threshold at $100 \mu \mathrm{V}$ for vertical and $40 \mu \mathrm{V}$ for horizontal components [10]. EEG channels are referenced to the common average and filtered in the alpha band $(8-14 \mathrm{~Hz})$ using a Butterworth band-bass filter (order 4). Alpha band oscillations are downsampled to $512 \mathrm{~Hz}$ and epoched $1.5 \mathrm{~s}$ before target display. Let $X \in \mathbb{R}^{C \times T}$ the resulting set of bandpassed signals with $C$ the number of electrodes and $T$ the number of time samples for each epoch.

\section{B. Feature Extraction}

We used a regularized version of the Common Spatial Pattern (CSP) algorithm [6], [4] to extract relevant features in the spatially filtered signals $X_{c s p} \in \mathbb{R}^{C \times T}$. The spatial filters $W=\left\{w_{j} \in \mathbb{R}^{C}\right\}_{j=1, \ldots, C}$ are computed by solving the generalized eigenvalue problem: $\Sigma_{l} W=\lambda \Sigma_{r} W$, with $\Sigma_{l}$ (resp. $\Sigma_{r}$ ) the covariance of $X$, for left (resp. right) trials. During online sessions, $X$ are projected from the original sensor space to the surrogate sensor space by $X_{c s p}=W^{T} X$. Finally, the band power features are defined as the log variance of the squared spatially filtered signals. Features are normalized to fit a normal distribution using the mean and the variance of features extracted in predictive trials.

\section{Classification}

In order to estimate the spatial location of attention, this BCI has to classify features of ambiguous trials as corresponding to left or right attention shifts. We train a linear SVM with features extracted and selected in the predictive condition using the Recursive Feature Elimination (RFE) procedure [5]. RFE recursively prunes and ranks features given the weights assigned by the SVM with a regularization parameter ranging from $10^{-5}$ to $10^{5}$. We select the feature set and regularization parameter that minimize the hinge loss of the SVM averaged over an inner 5-fold Cross Validation. This procedure runs on the HPC cluster for every trial recorded from the beginning of the experiment and updates the spatial filters, feature set and SVM parameters after each block.

The classification performance is evaluated with the percentage of true positives $\left(t_{p}\right)$, true negatives $\left(t_{n}\right)$, false positives $\left(f_{p}\right)$, and false negatives $\left(f_{n}\right)$. The proportion of relevant targets and warnings displayed by the BCI is evaluated with the accuracy, precision and recall score:

- Accuracy score : $\left(t_{p}+t_{n}\right) /\left(t_{p}+t_{n}+f_{p}+f_{n}\right)$

- Precision score : $t_{p} /\left(t_{p}+f_{p}\right)$

- Recall score : $t_{p} /\left(t_{p}+f_{n}\right)$ 


\section{Online Adaptation}

During the $1^{\text {st }}$ online session, the BCI displayed VT at the location identified by features classification (left vs right) for ambiguous trials. The classification accuracy is updated trial-by-trial based on the subject's report over the last 52 trials with ambiguous SC. The proportion of predictive $(\alpha)$ and ambiguous $(\beta) \mathrm{SC}$ are computed as a function of SER with predictive $\left(S E R_{\text {pred }}\right)$ and ambiguous $\left(S E R_{a m b i}\right)$ SC. Here, $S E R_{a m b i}$ is equal to BCI classification error since VT are displayed based on BCI classification. Assuming that subjects would strongly shift attention if the average SC identification rate is higher than $80 \%$ [8], the proportion of cues is adapted online by solving the equation:

$$
\begin{aligned}
& \alpha \times S E R_{\text {pred }}+\beta \times S E R_{\text {ambi }}=20 \% \\
& \alpha \times\left(100-S E R_{\text {pred }}\right)+\beta \times\left(100-S E R_{\text {ambi }}\right)=80 \%
\end{aligned}
$$

If $S E R_{a m b i} \leq 20 \%$, we set $\alpha=0$ and $\beta=100 \%$. For calibration, we expect $S E R_{\text {pred }}=10 \%$ and $S E R_{\text {ambi }}=50 \%$ from the threshold computed by the adaptive procedure and set $\alpha=75 \%$ and $\beta=25 \%$ by solving (1). For the $1^{\text {st }}$ online session, we initialize $S E R_{a m b i}$ from data recorded in calibration and update $\alpha$ and $\beta$ after each trial, keeping $S E R_{\text {pred }}=10 \%$ to prevent mixing factors.

For the $2^{\text {nd }}$ online session, the BCI warned the subject $140 \mathrm{~ms}$ before VT display by flashing its location when features were classified to the opposite location. We kept $\alpha$ and $\beta$ at their value of the last update of the $1^{\text {st }}$ online session, assuming classification performance would remain stable, even if $S E R_{a m b i}$ was higher than the previous sessions.

\section{RESULTS}

\section{A. Behavioral Performance in offline session}

\begin{tabular}{|c|c|cc||c|cc|}
\cline { 2 - 7 } \multicolumn{1}{c|}{} & \multicolumn{3}{c||}{ Predictive SC } & \multicolumn{3}{c|}{ Ambiguous SC } \\
\hline Subj. & SER & RT & ER & SER & RT & ER \\
\hline S1 & 13.46 & 0.662 & 12.18 & 48.08 & 0.722 & 23.08 \\
\hline S2 & 9.62 & 0.873 & 24.36 & 36.54 & 1.011 & 34.62 \\
\hline S3 & 19.23 & 0.837 & 21.79 & 34.62 & 1.045 & 25.00 \\
\hline S4 & 8.33 & 1.187 & 16.03 & 21.15 & 1.443 & 15.38 \\
\hline S5 & 7.05 & 0.833 & 16.67 & 42.31 & 0.940 & 15.38 \\
\hline Mean & $\mathbf{1 1 . 5 4}$ & $\mathbf{0 . 8 7 9}$ & $\mathbf{1 8 . 2 1}$ & $\mathbf{3 6 . 5 4}$ & $\mathbf{1 . 0 3 2}$ & $\mathbf{2 2 . 6 9}$ \\
\hline
\end{tabular}

TABLE I

\section{CALIBRATION RESULTS}

Table I shows the SER (\%), RT (s) and ER (\%) for predictive and ambiguous SC. On average, $3.5 \%$ of the trials were contaminated by eye movements and rejected from the analysis and $51.05 \pm 4.19 \%$ of VT were displayed at the right location. As expected, subjects made higher SER for ambiguous than predictive SC. However, SER for ambiguous $\mathrm{SC}$ in S2, S3 and S4 is significantly lower than chance level compared to a binomial distribution of 52 samples $(p<$ 0.05 ). This effect may be due to some implicit learning of the sequence of left and right target presentations. Nonetheless, RT and ER are on average 0.153 s shorter and $4.48 \%$ lower for predictive than for ambiguous SC. Indeed, RT and ER are enhanced when the VT is displayed at the attended location compared to the unattended one. Mean difference between attended and unattended VT is $-0.598 \mathrm{~s}$ in RT and $-46.19 \%$ in ER for predictive SC, and $-0.541 \mathrm{~s}$ in RT and $-36.69 \%$ in ER for ambiguous SC.

\section{B. Adaptive Target Session}

On average, $2.64 \%$ of trials contaminated by eye movements were rejected from the analysis. The ambiguous SC proportion $(\beta)$ is computed with (1) and adapted online trialby-trial as shown in Figure 1.

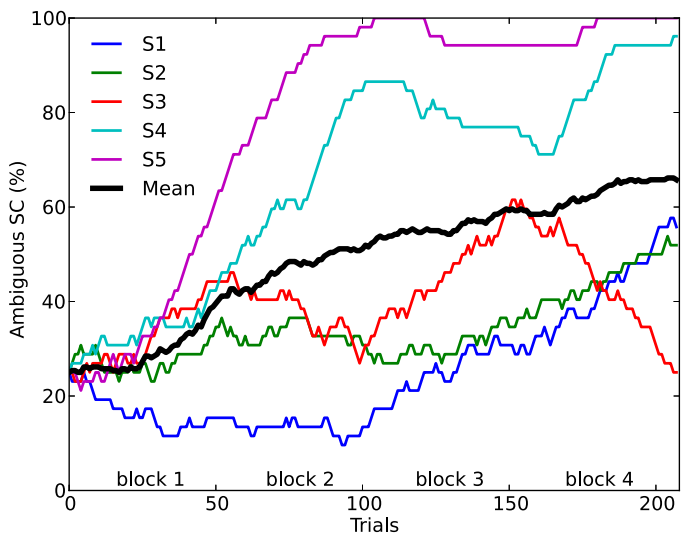

Fig. 1. Online adaptation of ambiguous SC

As expected, $S E R_{a m b i}$ is reduced when BCI display VT at a correctly classified location which increases the amount of ambiguous SC online, because of (1). This increase of classification accuracy is due to BCI training between each block with features extracted and selected from the beginning of the experiment. Nonetheless, online adaptation is quite variable across subjects and can increase after several blocks of trials (S1 and S2).

\begin{tabular}{|c|ccc||cc|cc|}
\cline { 2 - 8 } \multicolumn{1}{c|}{} & \multicolumn{3}{c||}{ Classification } & \multicolumn{4}{c|}{ Ambiguous SC } \\
\hline Subj. & Acc. & Prec. & Rec. & $\beta$ & SER & RT & ER \\
\hline S1 & 71.67 & 72.22 & 78.79 & 29.13 & 28.33 & 0.633 & 13.33 \\
\hline S2 & 63.29 & 71.88 & 53.49 & 38.16 & 36.71 & 1.128 & 24.05 \\
\hline S3 & 64.63 & 58.82 & 57.14 & 40.00 & 35.37 & 0.751 & 23.17 \\
\hline S4 & 88.82 & 94.59 & 84.34 & 75.62 & 11.18 & 1.017 & 11.84 \\
\hline S5 & 87.86 & 87.72 & 78.13 & 89.18 & 12.14 & 0.834 & 10.98 \\
\hline Mean & $\mathbf{7 5 . 2 5}$ & $\mathbf{7 7 . 0 5}$ & $\mathbf{7 0 . 3 8}$ & $\mathbf{5 4 . 4 2}$ & $\mathbf{2 4 . 7 5}$ & $\mathbf{0 . 8 7 3}$ & $\mathbf{1 6 . 6 8}$ \\
\hline
\end{tabular}

TABLE II

ADAPTIVE TARGET RESULTS

Table II shows the classification performance in terms of accuracy, precision and recall (\%) and behavioral performance for ambiguous trials in terms of $\beta$ parameter (\%), SER $(\%)$, RT (s) and ER (\%). As noted earlier, the SER is equal to the classification ER because the BCI displayed targets at classified location for ambiguous SC. On average across subjects, the proportion of ambiguous SC is $29.42 \%$ higher than during the calibration. The difference between attended vs unattended target is $-0.353 \mathrm{~s}$ in RT and $-37.01 \%$ in ER for ambiguous SC. However, SER is reduced by $11.79 \%$ for ambiguous SC and increased by $13.21 \%$ predictive SC, compared to calibration. Consequently, RT and ER are enhanced by $0.159 \mathrm{~s}$ and $6.02 \%$ for ambiguous SC. In addition, RT and ER difference between calibration and this 
online session for predictive SC remained stable with $5.63 \mathrm{~ms}$ and $-0.46 \%$.

\section{Adaptive Warning Session}

\begin{tabular}{|c|ccc||cc|cc|}
\cline { 2 - 8 } \multicolumn{1}{c|}{} & \multicolumn{3}{c||}{ Classification } & \multicolumn{4}{c|}{ Ambiguous SC } \\
\hline Subj. & Acc. & Prec. & Rec. & $\beta$ & SER & RT & ER \\
\hline S1 & 68.97 & 68.00 & 62.69 & 57.43 & 46.55 & 0.633 & 20.69 \\
\hline S2 & 75.47 & 86.36 & 65.52 & 50.96 & 54.72 & 0.688 & 30.19 \\
\hline S3 & 72.50 & 70.00 & 73.68 & 38.46 & 47.50 & 0.916 & 10.00 \\
\hline S4 & 75.73 & 50.00 & 92.00 & 100.00 & 24.27 & 0.888 & 9.71 \\
\hline S5 & 91.18 & 92.98 & 91.38 & 100.00 & 56.86 & 0.633 & 15.69 \\
\hline Mean & $\mathbf{7 6 . 7 7}$ & $\mathbf{7 3 . 4 7}$ & $\mathbf{7 7 . 1 1}$ & $\mathbf{6 9 . 3 7}$ & $\mathbf{4 5 . 9 8}$ & $\mathbf{0 . 7 5 2}$ & $\mathbf{1 7 . 2 5}$ \\
\hline
\end{tabular}

TABLE III

ADAPTIVE WARNING RESULTS

In this session, after classifing left vs right shifts of attention, the BCI no longer adapts the target location but it issues a warning by flashing its location if VT was predicted to be unattended. On average, $1.15 \%$ of trials contaminated by eye movements were rejected from the analysis. The classification and ambiguous performance are presented in table III. As we had assumed, classification performance is still good although SER is at chance level [10]. The average ambiguous SC proportion $(\beta)$ is $44.37 \%$ higher than calibration and $14.95 \%$ higher than the adaptive display session. The mean difference between attended vs unattended VT is $0.039 \mathrm{~s}$ in RT and $-19.63 \%$ in ER for ambiguous SC trials. Indeed, subjects responses are on average speeded for attended and unattended VT because of warnings displayed at target location. But the warning display also impaired VT identification for subjects S2, S3 and S5. Consequently, RT and ER are enhanced by $0.280 \mathrm{~s}$ and $5.44 \%$ in the session for ambiguous SC. Moreover, the difference between the offline and this online session for predictive SC are $0.165 \mathrm{~s}$ for RT and $1.74 \%$ for ER.

\section{DISCUSSION}

The paper presents a BCI for enhancing visuospatial attention performance of human subjects in a complex task. During the offline session, subjects reacted to spatially precued targets on average faster and more accurately with predictive than ambiguous cues. Behavioral enhancement and classification performance were evaluated over two consecutive online sessions. During the $1^{\text {st }}$ online session, targets were displayed at attended location by an active BCI based on endogenous attention. During the $2^{\text {nd }}$ online session, subjects were warned of spatial error before target display by a passive BCI based on exogenous attention.

In the online sessions, behavioral performance was enhanced for ambiguous SC and remained stable for predictive SC compared to the offline session. The BCI classification performance increased during the $1^{\text {st }}$ online session, enabling adaptation of the ambiguous SC proportion while reducing spatial errors. Interestingly, the behavioral and classification performance remained stable during the $2^{\text {nd }}$ online session although subjects had a high SER. Subjects were able to voluntarily control target display at each location during the $1^{s t}$ online session and received relevant warning to prevent impaired reaction to targets displayed at unattended locations during the $2^{\text {nd }}$ online session.

An implication for neuroscience research is new evidence about possible single trial classification, even when subjects make more than $20 \%$ of spatial errors [8]. This paper extends the potential of BCI technology by enhancing visuospatial attention performance through adaptive and warning displays. Neuroergonomics applications based on evoked potential reflecting human error [7] could be used to detect BCI errors and avoid subjective reports to record spatial error of target display. Moreover, EEG can reflect many covert processes including perception, emotion, reasoning and decision making capability of the operator. Future BCI applications could enhance the chance of success in critical situations by adaptive human-computer interactions through BCI.

\section{ACKNOWLEDGMENT}

The authors gratefully thank the subjects for participating in the experiment and M. Dyson for developping the EEG drivers for OpenViBE. This work was supported by the DGA and the CoAdapt ANR project (ANR-09-EMER-002).

\section{REFERENCES}

[1] A. Bahramisharif, M. van Gerven, T. Heskes, and O. Jensen, Covert attention allows for continuous control of brain-computer interfaces. Eur. J. Neurosci., vol. 31(8), 2010, pp. 1501-1508.

[2] A. Bahramisharif, T. Heskes, O. Jensen, and M. van Gerven, Lateralized responses during covert attention are modulated by target eccentricity. Neurosci. Lett., vol. 491(1), 2011, pp. 35-39.

[3] B.A. Dosher, and Z.L. Lu, Noise exclusion in spatial attention. Psychol. Sci., vol. 11(2), 2000, pp. 139-146.

[4] J. Fujisawa, H. Touyama, and M. Hirose, Extracting alpha band modulation during visual spatial attention without flickering stimuli using common spatial pattern. Conf. Proc. IEEE Eng. Med. Biol. Soc., 20-25 Aug. 2008, pp. 620-623.

[5] I. Guyon, J. Weston, S. Barnhill, and V. Vapnik, Gene selection for cancer classification using support vector machines. Mach. Learn. vol. 46, 2002, pp. 389-422.

[6] F. Lotte, and G. Guan, Regularizing Common Spatial Patterns to Improve BCI Designs: Unified Theory and New Algorithms. IEEE Trans. Biomed. Eng., vol. 58(2), 2011, pp. 355-362.

[7] R. Parasuraman, M. Rizzo, Neuroergonomics: The Brain at Work Oxford University Press, Inc., 2006.

[8] M. Posner, Orienting of attention. Quart. J. Exp. Psych., vol. 32(1), 1980, pp. 3-25.

[9] Y. Renard, F. Lotte, G. Gibert, M. Congedo, E. Maby, V. Delannoy, O. Bertrand, and A. Lécuyer Openvibe: An open-source software platform to design, test, and use brain-computer interfaces in real and virtual environments, Presence: Teleoper. Virtual Environ., vol. 9, Feb. 2010, pp. 35-53,

[10] T.A. Rihs, C.M. Michel, and G. Thut, A bias for posterior $\alpha$ band power suppression versus enhancement during shifting versus maintenance of spatial attention. NeuroImage, vol. 44(1), 2009, pp. 190-199.

[11] M. Treder, A. Bahramisharif, N. Schmidt, M. van Gerven, and B. Blankertz, Brain-computer interfacing using modulations of alpha activity induced by covert shifts of attention. J. NeuroEng. Rehab., vol. 8(1), 2011, pp. 24.

[12] M. van Gerven, and O. Jensen, Attention modulations of posterior alpha as a control signal for two-dimensional brain-computer interfaces. J. Neuro. Meth., vol.179(1), 2009, pp. 78-84.

[13] J.B.F. Van Erp, F. Lotte, and M. Tangermann, Brain-Computer Interfaces: Beyond Medical Applications. IEEE Computer, vol. 45(4), 2012, pp. 26-34.

[14] A. Watson, and D. Pelli, QUEST: a Bayesian adaptive psychometric method. Percept. Psychophys., vol 33(2), Feb. 1983, pp. 113-120. 